\title{
COVID-19: Simultaneous surveillance studies and case series, Jordan as a Case Study
}

\author{
Ahmad Khalaf Alkhawaldeh ${ }^{1, *}$ \\ ${ }^{1}$ University of Jordan, Department of Chemistry, Amman - 11942, Jordan. \\ *Corresponding Author E-mail: Ahm9140096@fgs.ju.edu.jo, Ahmad.alkawalda@yahoo.com \\ Phone: 00962787455167
}

\begin{abstract}
The outbreak of coronavirus 2 (SARS-CoV-2) has already taken on pandemic proportions and has affected more than 100 countries in recent weeks. It is imperative that global healthcare systems are prepared. Containment initiatives in Jordan, on the other hand, have decreased new cases by over 63\%. The Jordanian national health system has been very pleased with its ability to adequately meet the needs of the patients diagnosed with SARS-CoV-2 pneumonia, and includes intensive care. From 2 March to 14 April 2020, between 4\% and 8\% of active infected patients in Jordan registered daily in intensive care. The percentage is very low.
\end{abstract}

Keyword: COVID-19, coronavirus, SARS-CoV-2, Simultaneous surveillance and Jordan.

\section{Introduction}

The recent outbreak of viral pneumonia in China was the result of a novel coronavirus. The spread of coronavirus disease (COVID-19) in the year 2019 has already been demonstrated by over 100,000 persons in 100 countries and the epidemiological demands needed for a pandemic disease has already been met [1]. The latest 2019 outbreak of coronavirus (now known as SARS-CoV-2 causing the Covid-19 disease) has spread from Wuhan to a variety of countries, some of which have continuously transmitted. Modern attempts were made to identify the clinical course, report serious cases and treat ill people. Experiences with Middle East respiratory syndrome (MERS), influenza pandemics and other outbreaks have shown that we face the urgent need to increase the public health efforts in order to explain and classify the novel virus epidemiology. The effect of an epidemic depends on the number, transmissibility of the virus, and clinical seriousness of the epidemic [2]. Therefore, we need a concerted global response to prepare healthcare systems to meet this unparalleled challenge. Paradoxically, countries poor enough to be vulnerable to the outbreak still have important lessons to recall. Although new cases in China have decreased by over $90 \%$ at present, this is not the case in other countries, including Italy and Iran [3]. In order to calibrate the outbreak response, the number of cases must be calculated, including mild. Conventional wisdom allows sickest individuals to seek treatment and undergo testing; the effect is also measured early in an outbreak with case fatality and hospitalization ratios. Such steps should be taken with caution as it can take time for extreme cases to occur, or for those infected to die, 
and the denominator of infected people to ratio such figures may not be correctly calculated. As was the case for previous epidemics, first cases of Covid-19 in China were sufficiently severe to be checked and medical treatment, but the overall number of persons infected was negligible. The average fatal ratio of patients seeking medical attention is up to now around $2 \%$, but for a while the true ratio is not understood [4]. The average age of death was 81, and for more than two-thirds of these patients, diabetes, medical disorders and/or cancer have or have been former smokers. It should also be remembered that these patients had specific health conditions, but also had acute respiratory distress syndrome (ARDS) and severe acute coronavirus syndrome (SARS-CoV-2) pneumonia that needed respiratory support and would probably not have died as pneumonia (SARS -CoV-2) has high mortality rates and survival levels. Pneumonia has a high (SARS -CoV2) mortality rate [5]. Simple counts of confirmation numbers may be inaccurate measures of the course of the outbreak when such counts are restricted by issues in laboratory test access to care or bottlenecks or if only severe cases have been identified. Throughout the 2009 flu pandemic, an approach to tracking where cases are too frequent to count was identified. This method, adaptable to Covid-19, includes the use of existing surveillance systems or the planning of weeksly checking for the novel coronavirus of a subset of certain individuals, in order to find out the number of people with extremely susceptible but not particular syndrome (e.g. acute respiratory infection) every week. The consequence of the occurrence of acute respiratorial infections (for example) and the percentage of positive testing provides an indication of the burden of case in a given jurisdiction. The performance of these and other viral testing public health research can be greatly enhanced by viral testing [6]. More broadly, combined monitoring, epidemiological fieldwork and case series are useful for synthesizing data. Cohort studies in well-identified settings like schools, workplaces, or neighbourhoods can better explain the overall burden and the incidence of attacks on the household and the community; even more significantly, they can easily measure the magnitude of the epidemic by counting the number of health, hospital and deaths in a given population and extrapolates. Transmissibility awareness remains essential to the prediction of the trajectory of the outbreak and the possibility that transmission can proceed. Several groups estimated SARS-CoV-2 reproductive $\mathrm{R}_{0}$ with epidemic curves, but household studies can be superior data source on the transmission timing and likelihood, and can help to estimate $\mathrm{R}_{0}=0.5$ [7]. The Government of Jordan has adopted exemplary steps that reduce the risk of contact with non-infected persons, to limit viral transmission. It is undoubtedly a significant and brave decision.

\section{Modelling predictions}

The following forecasts are provided in preparation for what will happen in the coming days and weeks for our political leaders - people with the greatest responsibility for national health systems and state government, and local health authorities. You will then take steps to address the demands of this tough time with medical personnel and hospital beds. The big question at this stage is whether the number of patients being infected will continue to rise exponentially and for how long. The only way to predict these kinds of problems is by analyzing the patterns of COVID-19 infection data collected by the Jordanian population. 


\section{Public Health and Social Measures for the COVID-19 Pandemic in Jordan:}

The intervention of people, organisations, societies, local and national governments, and the international bodies to delay or avoid the implementation of COVID-19 shall include public health and social initiatives. Other steps to minimize COVID-19 spread include person and environmental interventions, cases of identification and separation, touch monitoring and quarantine, steps for the distancing of social and physical, including controls on mass collections and international travel. Social and physical distancing steps are aimed at slowing down the spread of the disease by avoiding the emergence of new chains of COVID-19 transmission. These initiatives protect physical distance (minimum one meter) between individuals, minimize interaction with polluted areas, promote, and maintain virtual social links between families and communities, respectively. The steps to be followed by citizens include the implementation of flexible working arrangements such as telework, distance learning; the elimination and avoidance of overcrowding; the closedown of non-essential facilities and services; the protecting and security of disadvantaged groups. The measures are used along with individual COVID-19 preventive steps including regular washing of the hand and cough etiquette. The organization of work sites to ensure physical distance between individuals, such as shifts over time and the on-site transfer of service to home delivery will help keep more companies accessible. Tele-working and tele-schooling approaches demonstrate creativity and the role of technology in promoting business continuity and social relations in families and societies in various contexts. Generally, the introduction of distancing steps should also aim at preserving personal and professional relations by means of interactive means and technology.

\section{Results}

During a COVID-19 virus outbreak, official numbers of infected individuals in Jordan reflect the spread of the disease; since 2 Mar 2020, the number of patients infected has been reported every day. Data from up to 14 April indicates that the trend in previous days, reported cases, new cases, total deaths, and total recoverable and active cases showed an increase in patient infection. As Figure 1 reveals. Total cases in all cities in Jordan are shown in Figure 2. 


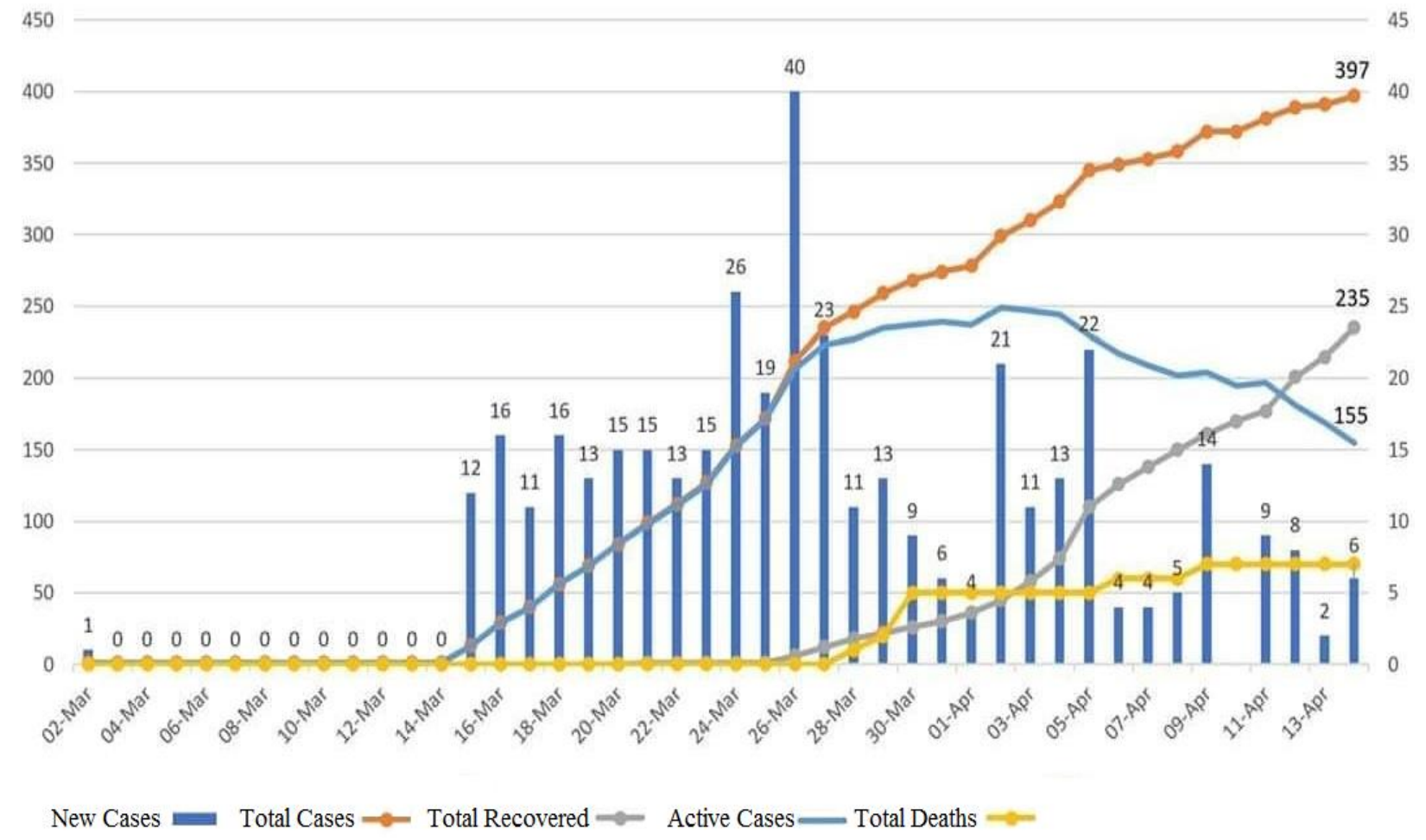

Figure 1: Number of infections in previous days, total cases, new cases, total deaths, total recovered and active Cases at Apr 14 in Jordan.

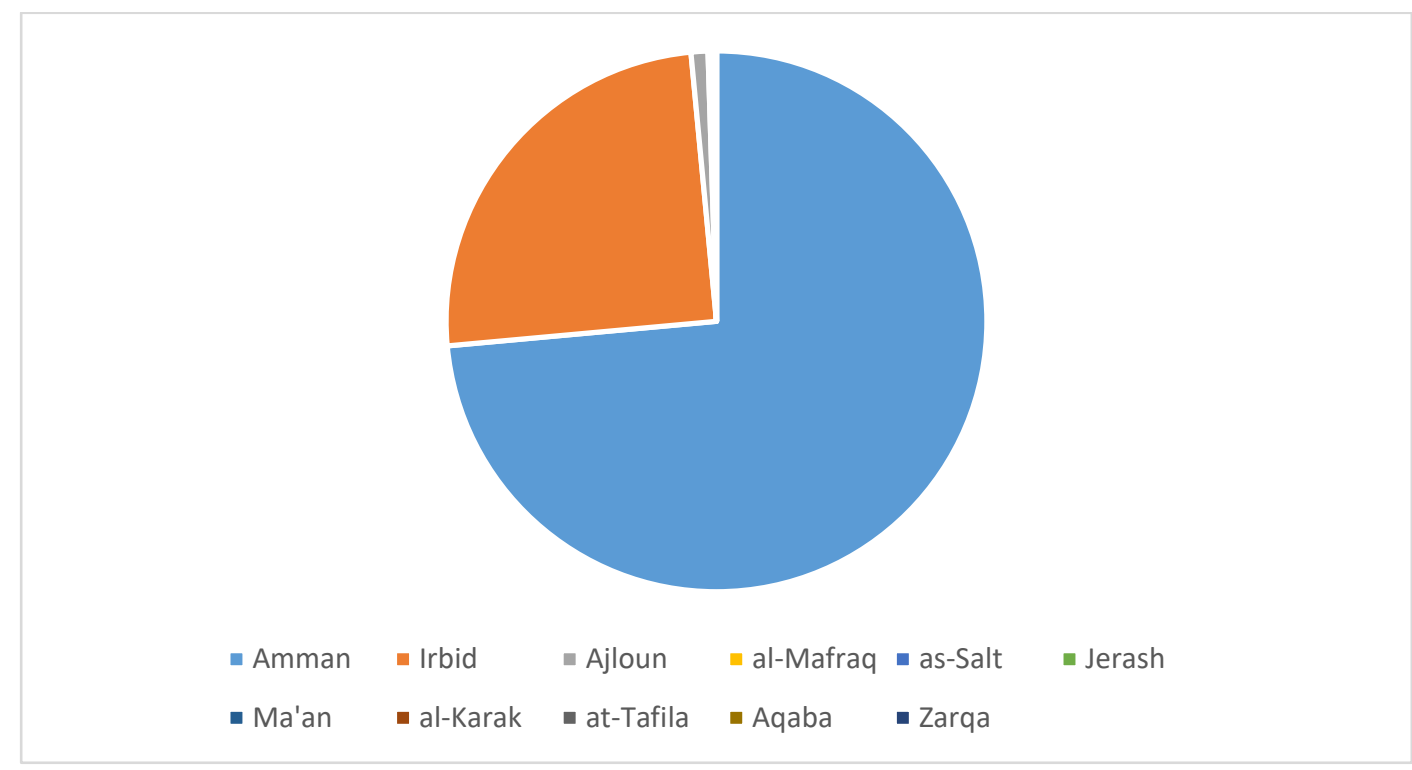

Figure 2: Distribution of total cases in all city in the Jordan. 


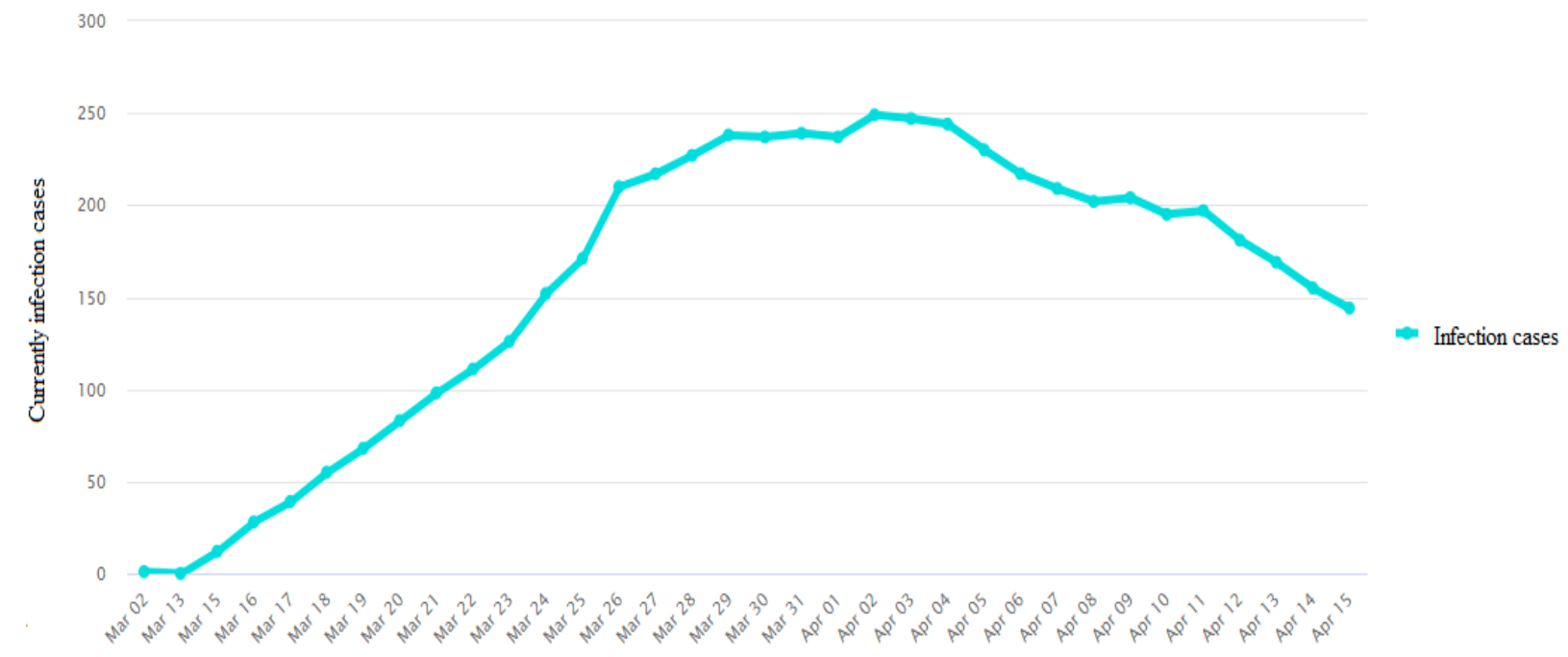

Figure 3: The number of patients reported to be infected and recovered in Jordan through 14 April 2020.

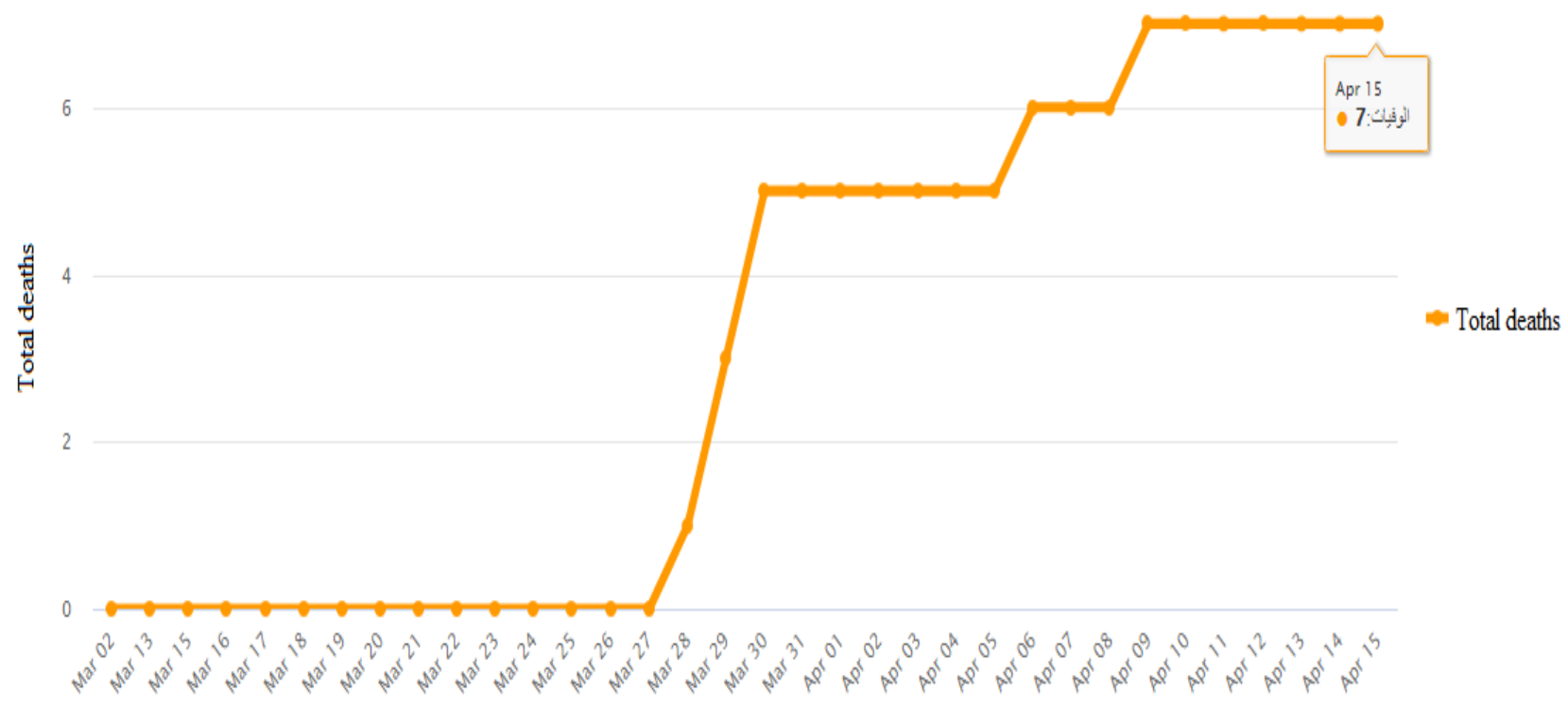

Figure 4: laboratory-confirmed COVID-19 cases and deaths. Data as of 14 April 2020 


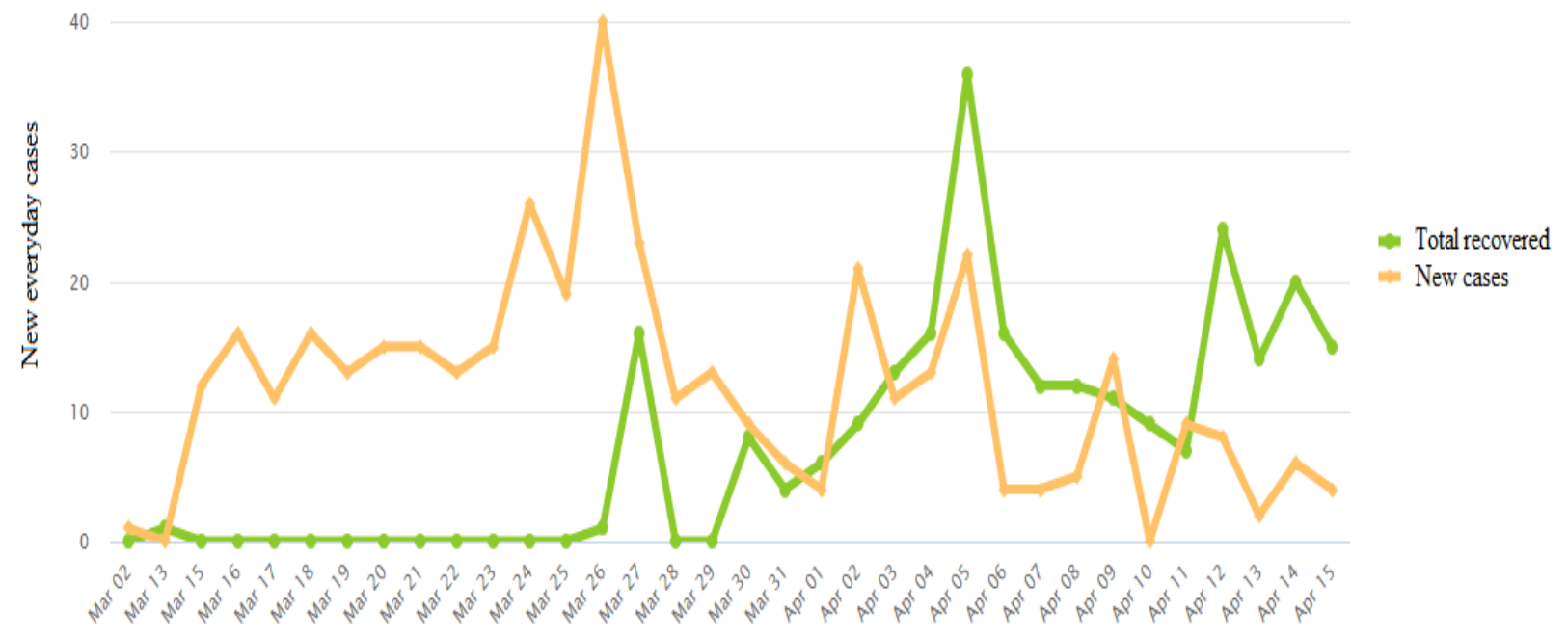

Figure 5: The number of patients reported to be infected and total recovered in Jordan using an exponential curve.

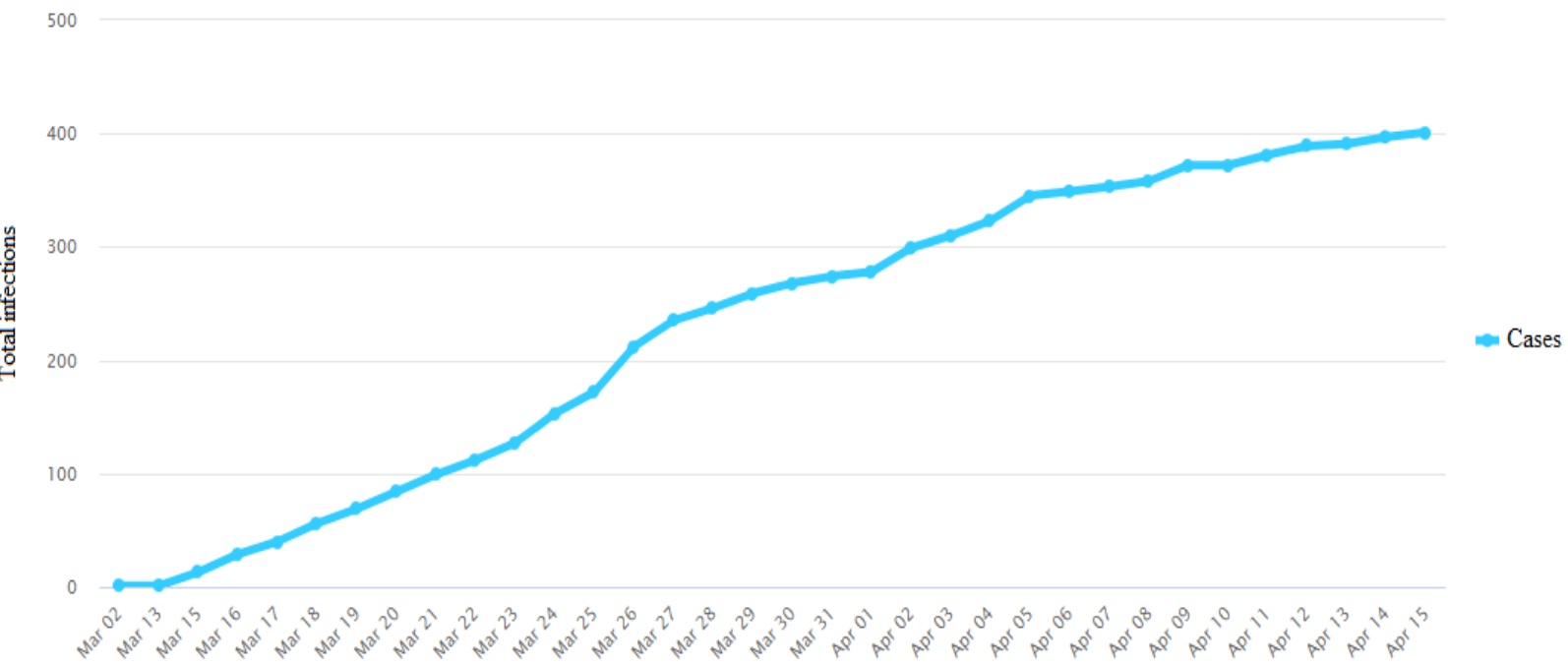

Figure 6: Epidemic curve of confirmed COVID-19 in Jordan through 14 April 2020.

The initial phase of an infection outbreak followed the predicted exponentially moving pattern, as shown in Figure 1. The number of infections per day rose steadily; in the history of 26 March: 40 new cases were reported as the largest number. The number of cases reported was higher than the number of new cases after 11Apr. By the end of April 14, 397 cases totalled and 235 recovered cases was recovered to a rate of $60 \%$. Amman and Irbid concentrated most of the infections. In the 
capital Amman, the main distribution of infections was $73.9 \%$ and then Irbid $25 \%$. There were significant rises in the number of patients worldwide, with an exponential rise up to 16 April. Data from Jordan's Health Ministry can best be obtained using the same exponent as the number of infected patients, as shown in Figure 6. From Mar 15, 17 days after the exponential increase, the total number of the infected patients began to diverge; we propose that, within 3-4 days of April, the number of newly infected patients will begin to decline.

\section{Discussion}

A family cluster of 5 COVID-19 patients had contact with an asymptomatic family member returning from the Wuhan outbreak in Anyangs, China before the onset of symptoms [8]. The sequence of events shows the asymptomatic carrier will transmit the coronavirus. For patient 1 , the incubation period was 19 days, but was stated to be 0 to 24 days [9]. The first result of their RT-PCR was negative; the consistency of the kit, samples obtained or the output of the test was found to be false negative. For diagnostic virology, RT-PCR has been widely used and shows few false positive tests. Therefore, her second RT-PCR findings were unlikely and were used to characterize a coronavirus infection causing COVID-19 [10]. Further study is needed for asymptomatic carriers to acquire and transmit COVID-19 coronavirus [11]. Furthermore, the current approach to these patients in jordan includes non-pharmacological, pharmacological and antiretroviral treatments that may lead to distortion of calculations. We have no conclusive proof at this moment. As more precise forecasts, we should find the exact number of patients infected in coming days or weeks. According to the available evidence, at 14 April 2020 in Jordan, the number of infected patients reached about 400, as the new cases dropped to almost zero. As the number of patients who are deliberately diagnosed with ARDS currently stands at approximately 5\%. Electrode Nanostructures used COVID-19 Control and detection [12- 18].

\section{Conclusion}

We are in principle better positioned to respond to the current outbreak than many other countries. Nonetheless, an proactive approach has to be taken with SARS-CoV-2, also involving ventilation assistance patients who are severely ill. The program has been under immense pressure to adapt to changing circumstances. Such steps are a step in the right direction. In an unforeseen war on a formidable enemy, our doctors and nurses are modern heroes. They won't have any options in the near future. You would be expected to comply with the same requirements as health staff. Finally, our study indicates that steps to minimize transmission, as our government did on 9 March, will undoubtedly be enforced, unless specifically necessary, to stop the movement of people and social activities. 


\section{References}

1. Callaway, E. (2020). Time to use the p-word? Coronavirus enters dangerous new phase. Nature. doi: 10.1038/d41586-020-00551-1

2. Iacobucci, G. (2020). Covid-19: UK deaths are higher than previously reported, new data suggest. Bmj, m1330. doi: 10.1136/bmj.m1330

3. Carleton, T., \& Meng, K. C. (2020). Causal empirical estimates suggest COVID-19 transmission rates are highly seasonal. doi: 10.1101/2020.03.26.20044420

4. Lachmann, A., Jagodnik, K. M., Giorgi, F. M., \& Ray, F. (2020). Correcting under-reported COVID-19 case numbers: estimating the true scale of the pandemic. doi: 10.1101/2020.03.14.20036178

5. Strielkowski, W. (2020). International Tourism and COVID-19: Recovery Strategies for Tourism Organisations. doi: 10.20944/preprints202003.0445.v1

6. Wise, J. (2020). Covid-19: Death rate in England and Wales reaches record high because of covid-19. Bmj, m1484. doi: 10.1136/bmj.m1484

7. Lytras, T., Panagiotakopoulos, G., \& Tsiodras, S. (2020). Estimating the ascertainment rate of SARS-CoV-2 infection in Wuhan, China: implications for management of the global outbreak. doi: 10.1101/2020.03.24.20042218

8. Yu, P., Zhu, J., Zhang, Z., \& Han, Y. (2020). A Familial Cluster of Infection Associated With the 2019 Novel Coronavirus Indicating Possible Person-to-Person Transmission During the Incubation Period. The Journal of Infectious Diseases. doi: 10.1093/infdis/jiaa077

9. Lin, H., Liu, W., Gao, H., Nie, J., \& Fan, Q. (2020). Trends in Transmissibility of 2019 Novel Coronavirus-Infected Pneumonia in Wuhan and 29 Provinces in China. SSRN Electronic Journal. doi: 10.2139/ssrn.3544821

10. Hao, W., Li, M., \& Huang, X. (2020). First atypical case of 2019 novel coronavirus in Yanan, China. Clinical Microbiology and Infection. doi: 10.1016/j.cmi.2020.02.011

11. Wang, D., Hu, B., Hu, C., Zhu, F., Liu, X., Zhang, J., Peng, Z. (2020). Clinical Characteristics of 138 Hospitalized Patients With 2019 Novel Coronavirus-Infected Pneumonia in Wuhan, China. Jama, 323(11), 1061. doi: 10.1001/jama.2020.1585

12. Alshamaileh, E., Al-Sulaibi, M., Al-Khawaldeh, A., Almatarneh, M., El-Sabawi, D. and Al-Rawajfeh, A. (2016), "Current status of nanotechnology in Jordan", World Journal of Science, Technology and Sustainable Development, Vol. 13 No. 2, pp. 66-81. https://doi.org/10.1108/WJSTSD-01-2016-0001

13. Hourani, M. K. and Alkawaldeh A. (2016). Synergistic Effects of Bismuth Adatoms on Electrocatalytic Properties of Electrodeposited Nanostructured Platinum Electrodes. International Journal of Electrochemical Science, 3555-3566. doi: 10.20964/110434

14. Krishan, M.; Alkhawaldeh, A.; Soliman, A. (2019). Development of Nitride-Sensors for Monitoring in Control Systems. Preprints, 2019050227.

15. Almatarneh, M. H., Elayan, I. A., Al-Sulaibi, M., Khawaldeh, A., Saber, S. O. W., AlQaralleh, M., and Altarawneh, M. (2019). Unimolecular Decomposition Reactions of 
Propylamine and Protonated Propylamine. ACS Omega, 4(2), 3306-3313. doi: 10.1021/acsomega.8b02792.

16. Altweiq, A. and Alkhawaldeh, A (2019). The Determination of Some Heavy Metals in Different Selected Diets. Eurasian Journal of Analytical Chemistry, 14(4), emEJAC-00326. 17. Alkhawaldeh, A. K., M.Krishan, M., Altwaiq, A., Dabaibeh, R. N. (2020). Preparation of Nanostructured/ Microplatinum Surfaces by Application of a Square Wave Potential Regime for Methanol Oxidation. Eurasian Journal of Analytical Chemistry, 15(1), emEJAC-00362.

18. Al-Khedher, M. A., Pezeshki, C., Mchale, J. L., \& Knorr, F. J. (2012). Empirical Modeling of Nanoindentation of Vertically Aligned Carbon Nanotube Turfs using Intelligent Systems. Fullerenes, Nanotubes and Carbon Nanostructures, 20(3), 200-215. doi: 10.1080/1536383x.2010.542590 